\title{
Diffusion MRI Findings in Monochorionic Twin Pregnancies after Intrauterine Fetal Death
}

\author{
C. Hoffmann, B. Weisz, Y. Yinon, L. Hogen, L. Gindes, A. Shrim, E. Sivan, E. Schiff, and S. Lipitz
}

\begin{abstract}
BACKGROUND AND PURPOSE: Monochorionic twin pregnancies complicated by the IUFD of 1 twin are associated with substantial morbidity to the survivor twin. The aim of this study was to determine whether fetal sonography, T2 MR imaging, and DWI can diagnose acute cerebral lesions in the survivor of an MC twin pregnancy shortly after fetal death of the co-twin.
\end{abstract}

MATERIALS AND METHODS: During the study period (2007-2010) 34 cases of single IUFD were evaluated. Group A included 6 cases complicated by spontaneous IUFD. Group B had 10 cases of fetal death shortly after treatment of severe TTTS. These were compared with group C, with 18 pregnancies treated by selective termination due to severe complications in MC pregnancies.

RESULTS: Altogether 9/34 patients had abnormal prenatal cerebral findings. In group A, in 2/6 of pregnancies with spontaneous death, MR imaging showed findings of severe cerebral infarct, while cerebral damage was not evident by sonography. In another case, the surviving fetus was found to be hydropic on sonography, while MR imaging findings were normal. In group B, in 1/10 cases, cerebral infarct was demonstrated only by DWI. In 2 other cases, sonographic findings were normal, but MR imaging showed germinal matrix bleeding. In group C, in 1/18 cases, only DWI showed bilateral cerebral ischemia. In 2 other cases, MR imaging findings suggested germinal matrix bleeding and focal changes in the basal ganglia. In both cases, fetal sonographic findings were normal.

CONCLUSIONS: In our study, early manifestations of cerebral ischemia in monochorionic twin pregnancies were better diagnosed with MR imaging, especially with DWI.

ABBREVIATIONS: IUFD = intrauterine fetal death; MC = monochorionic; RFA = radio-frequency ablation; TTTS = twin-to-twin transfusion syndrome; US = sonography

M onochorionic twin pregnancies have a much higher risk of adverse outcome than their dichorionic counterparts because of vascular anastomoses that connect the 2 circulations. This shared circulation may lead to some unique complications, such as TTTS, twin anemia-polycythemia sequence, selective intrauterine growth restriction, and death or neurologic damage of the surviving twin if the co-twin dies during pregnancy. After a single fetal death, monochorionic twins are about 5 times more likely to have neurodevelopmental morbidity compared with di-

Received January 25, 2012; accepted after revision April 10.

From the Departments of Obstetrics and Gynecology (B.W., Y.Y., L.H., L.G., A.S., E.Sivan, E.Schiff, S.L.) and Radiology (C.H.), Sheba Medical Center, Tel-Hashomer Israel; Sackler School of Medicine (B.W., L.G., E.Sivan, E.Schiff, S.L.), Tel-Aviv University, Tel-Aviv, Israel; and Department of Obstetrics and Gynecology (B.W.), Sheba Medical Center, Talpiot Medical Leadership Program, Tel-Hashomer, Israel.

C. Hoffmann and B. Weisz contributed equally to this work.

Please address correspondence to Boaz Weisz, MD, Department of Obstetrics and Gynecology, Sheba Medical Center, Talpiot Medical Leadership Program TelHashomer, Israel; e-mail: boazmd@zahav.net.il

三 Indicates article with supplemental on-line table.

http://dx.doi.org/10.3174/ajnr.A3279 chorionic twins. ${ }^{1}$ The likely mechanism of brain damage is thought to involve acute hemodynamic imbalance due to exsanguination of the survivor into the dead fetus just before or at the time of fetal death. ${ }^{2,3}$ Some hypothesize that this results in intracranial hypoperfusion of the surviving twin, leading to cerebral ischemic insults. ${ }^{4,5}$

In some cases of single-twin death, intracranial abnormalities can be detected sonographically. ${ }^{6}$ However, the exact timing of the developing intracranial pathology in relation to the death of a co-twin has not been established. The role of MR imaging in diagnosing fetal cerebral abnormalities after the death of a co-twin is still unknown. A recent study has shown that a third of the surviving twins had abnormal findings on in-utero cerebral MR imaging, mostly undiagnosed by prenatal sonography. ${ }^{7}$ However, both prenatal sonography and conventional T2 MR imaging might fail to detect brain lesions in the first 2 weeks after the death of the co-twin. In the event of a cerebral ischemic lesion, diffusion of water molecules within the tissue is hindered by the swelling of cells and restriction of extracellular space, which are associated with the development of cytotoxic edema. A methodologic study ${ }^{8}$ 
and clinical case reports ${ }^{9-13}$ have confirmed the ability of DWI to detect acute cerebral ischemic lesions even during fetal life.

The aim of this study was to evaluate whether fetal sonography, T2 MR imaging, and DWI can diagnose acute cerebral lesions in the survivor of an MC twin pregnancy shortly after fetal death of the co-twin.

\section{MATERIALS AND METHODS}

This was a prospective cohort study of all cases of single fetal death (IUFD) in MC pregnancies analyzed by fetal sonography, T2 MR imaging, and DWI during a 4-year period (2007-2010) at Sheba Medical Center. Monochorionicity was based on first-trimester sonography. During the study period, 34 cases of single IUFD were evaluated. In group A, 6 cases were complicated by spontaneous IUFD. In group B, there were 10 cases of fetal death shortly after treatment of severe TTTS by laser ablation of communicating anastomoses. These were compared with group C, with 18 pregnancies treated by selective termination (bipolar or radiofrequency) due to severe complications in MC pregnancies.

Patients with spontaneous fetal death of a monochorionic pregnancy were hospitalized for evaluation of the surviving cotwin. According to our institutional protocol, a detailed sonographic scan (GE Voluson expert E8; GE Healthcare, Milwaukee, Wisconsin) was obtained shortly after the diagnosis of fetal death to detect cerebral lesions and fetal anemia. Fetal MR imaging was performed 24-72 hours after hospitalization, all within 6 days after fetal death. Patients after selective termination (either by bipolar coagulation or radio-frequency) had a detailed US scan and fetal MR imaging performed within 3 days after the procedure. Thereafter, all patients were scanned on a weekly basis by specialists in fetal medicine either after the diagnosis of IUFD (group A) or after interventions (groups B and C).

According to our MR imaging protocol, the mothers refrained from eating or drinking fluids with sugar 4 hours before the MR imaging examination, which was performed without sedation. Scans were obtained by using a 1.5T MR system (GE Healthcare). Singleshot fast spin-echo T2-weighted sequences in 3 orthogonal planes were used with section thickness of 3-4 $\mathrm{mm}$, with no gap, with a flexible coil (8-channel cardiac coil). The FOV was determined by the size of the fetal head and was $24 \mathrm{~cm}$ for smaller fetuses and $\leq 30 \mathrm{~cm}$ for the larger fetuses. Other parameters were a matrix of $320 \times 224$, a TE of $90 \mathrm{~ms}$, and a TR of $1298 \mathrm{~ms}$. The fast-spoiled gradient recalled T1 sequence was performed only in the axial plane with a larger FOV of $40 \mathrm{~cm}$, with a $4-\mathrm{mm}$ section thickness and a $0.5-\mathrm{mm}$ gap, a TR of $160 \mathrm{~ms}$, and a TE of $2.3 \mathrm{~ms}$. Then a DWI sequence in 3 orthogonal planes was performed with a $40-\mathrm{cm}$ FOV, b-values of 0 and 1000 or $700 \mathrm{~ms}$, and a section thickness of $4 \mathrm{~mm}$ with no gap. The ADC calculation map was added.

Patients were counseled about the potential risks for the surviving twins, including a substantial risk for neurologic abnormalities. Patients were informed that not all abnormalities can be diagnosed prenatally both by prenatal sonography or MR imaging. According to national abortion legislation, patients were able to elect termination of pregnancy after approval of a local committee.

Follow-up information was obtained from the charts of the hospital and from telephone interviews of the parents.

\section{RESULTS}

Thirty-four patients (all MC pregnancies with recent single fetal death) were examined by detailed US, DWI, and T2 MR imaging. Sixteen patients were examined after unintended fetal death $(6$ spontaneous IUFDs and 10 cases of single fetal death following treatment of TTTS by laser ablation), and 18 cases were evaluated after selective reduction. Altogether 9/34 patients had abnormal prenatal cerebral findings.

Group A: Spontaneous IUFD $(n=6)$. In $2 / 6$ pregnancies with spontaneous death, DWI showed severe findings of acute ischemic lesions (On-line Table, cases A.1 and A.2; Fig 1A, -B), while cerebral damage was not evident by sonography in either case (Doppler evaluation suggested fetal anemia in case A.1 due to elevated peak systolic velocity in the MCA). In case A.1, edema was demonstrated by the T2 sequence. In another case of IUFD at 25 weeks' gestation (case A.3), the surviving fetus was hydropic on US examination (ascites and thickened fetal skin), and fetal MCApeak systolic velocity levels $(56 \mathrm{~cm} / \mathrm{s})$ suggested fetal anemia. Initial DWI and T2 sequence findings were normal. Intrauterine blood transfusion was performed. The initial hematocrit level was $22 \%$ and was raised by packed-cell infusion to $42 \%$. A second MR imaging and follow-up scans showed no signs of cerebral damage. Findings on sonographic follow-up, including serial MCA-peak systolic velocity measurements, were normal throughout pregnancy. This patient delivered at 37 weeks' gestation, and the neonatal brain US findings were normal. Three other patients with spontaneous IUFD had normal prenatal US and MR imaging findings. Of those, 1 elected to have termination of pregnancy (against the departmental advice). Another patient delivered at 27 weeks due to placental abruption, and the child had sequelae of prematurity and asphyxia. The third patient delivered at 37 weeks' gestation, and the child is healthy without any neurologic sequelae.

Group B: Laser Ablation $(n=10)$. In $1 / 10$ cases complicated by IUFD after laser treatment, MR imaging showed findings of severe temporal lobe and periventricular infarcts to the co-twin (case B.1). This was seen only by DWI, but the parents elected to have termination of pregnancy. In another case, laser was performed at 21 weeks of gestation, and immediate DWI and T2 MR imaging findings were normal. Five days after the procedure, the "donor" twin died in utero. Sonographic examination findings of the co-twin were normal, but DWI showed germinal matrix bleeding, and the patient elected to have termination of pregnancy (case B.2; Figs $1 C,-D$ ). Another patient who had laser ablation at 24 weeks had germinal matrix bleeding seen initially only by DWI, and this was confirmed by T2 MR imaging at a following examination at 27 weeks' gestation (B.3). Seven other patients in group B without prenatal findings of brain injury delivered children without any neurologic sequelae.

Group C: Selective Reduction $(n=18)$. Selective termination was performed by bipolar coagulation $(n=11$; median gestational age, 21 weeks; range, $17-30$ weeks) or RFA ( $n=7$; median gestational age, 25 weeks; range, $22-32$ weeks). In 1/18 cases treated by selective termination, DWI showed bilateral cerebral ischemia 1 day after the procedure. T2 MR imaging findings at that point 


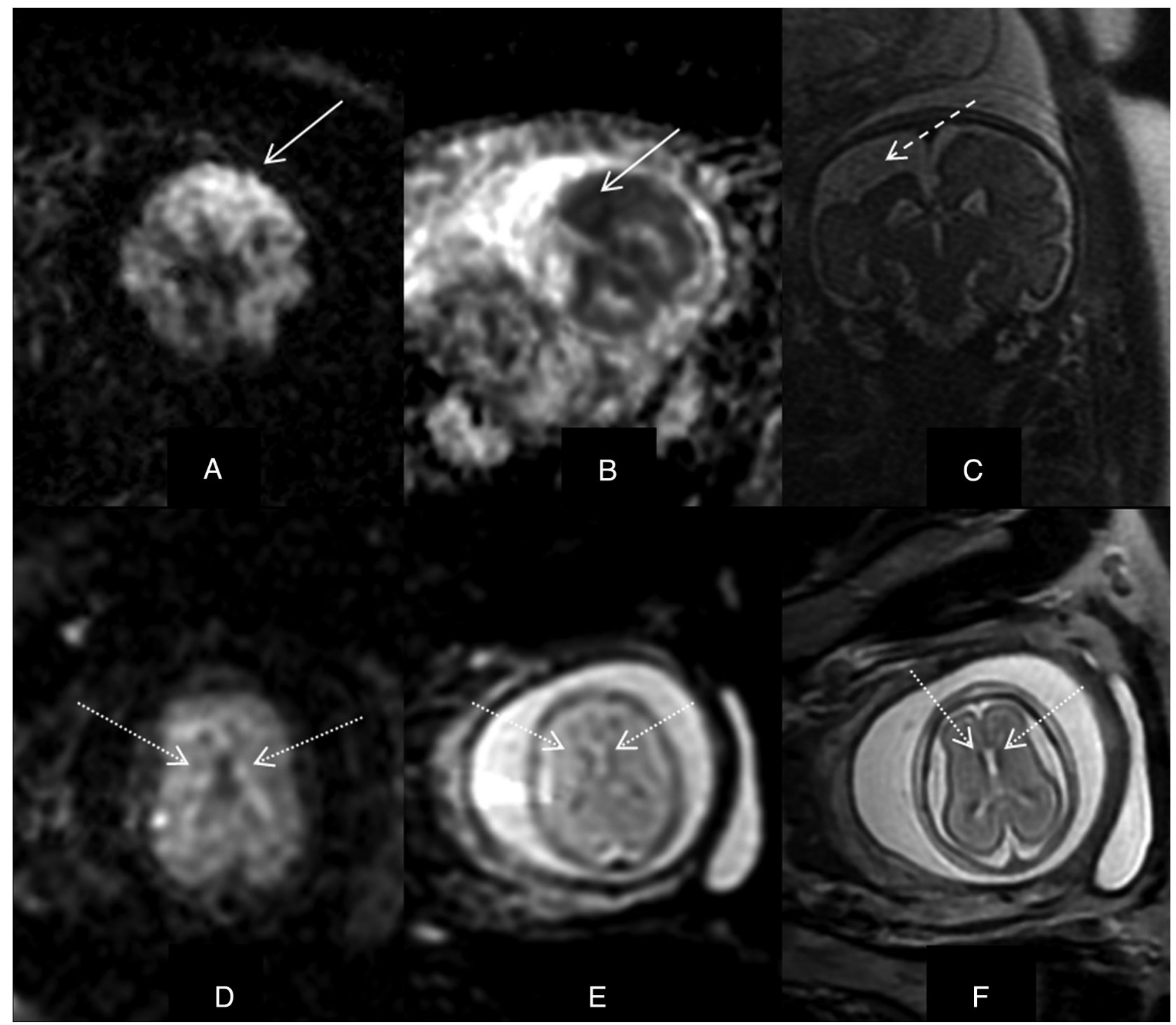

FIG 1. Diffusion MRI (dMRI) and T2 MR imaging following spontaneous fetal death (case A.2). dMRI ( $A$ ) and ADC image (B) show a unilateral recent infarct (solid arrow), while T2 MR imaging shows an unknown previous infarct (C, dashed arrow). Fetal death after laser ablation (case B.2). dMRI and ADC map show germinal matrix bleeding ( $D$ and $E)$, while T2 MR imaging shows bilateral periventricular hypointense signal $(F$, dotted arrow).
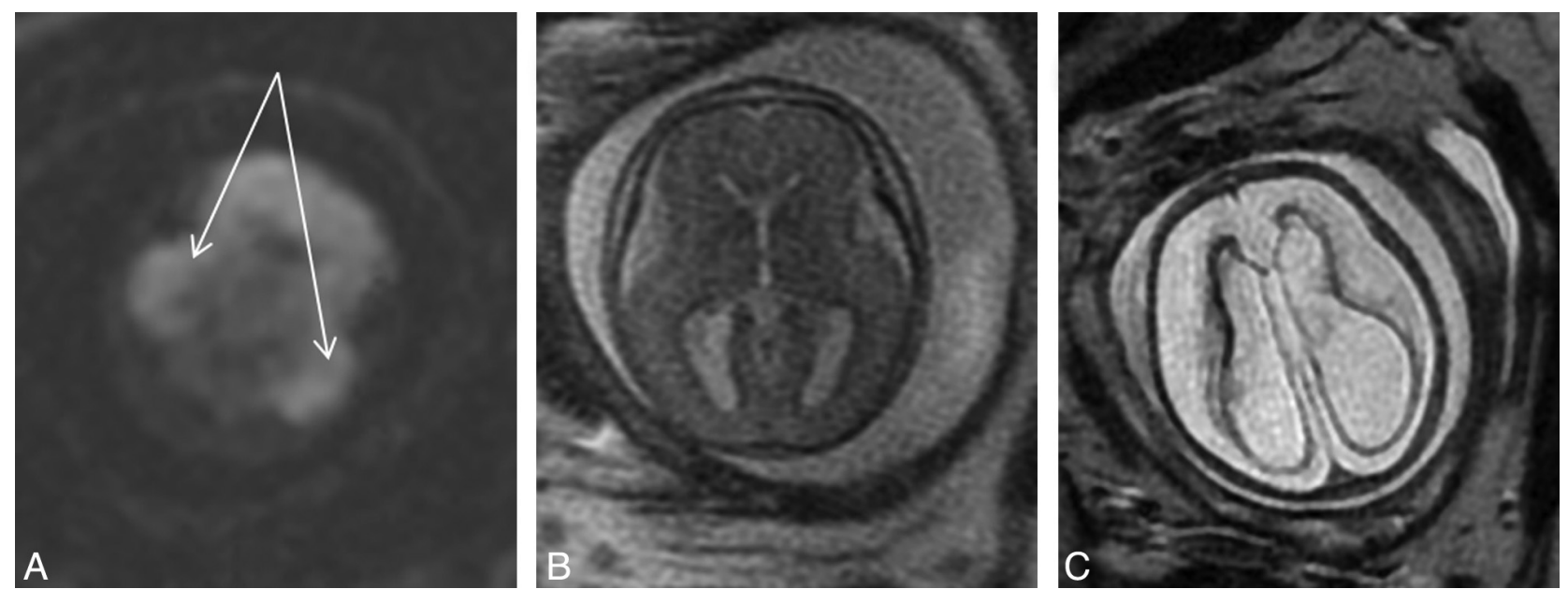

FIG 2. MR imaging findings 1 day after selective reduction showing bilateral cerebral ischemia by DWI (A, solid arrow), while T2 MR imaging findings were normal (B). Three weeks later T2 MR imaging shows hydroencephaly due to bilateral cerebral ischemia (C). 
were normal, and fetal brain injury was evident by T2 MR imaging only 3 weeks later (case C.1, Figs 2). The patient chose to have termination of pregnancy at 26 weeks' gestation. In 2 other cases, MR imaging findings suggested germinal matrix bleeding (case C.2) and focal changes in the basal ganglia (case C.3). In both cases, fetal sonographic findings were normal, both continued the pregnancies, and the children are neurologically intact at 1.5-2.5 years of age. Fifteen other neonates without prenatal signs of brain injury were delivered at term and are all alive and healthy.

\section{DISCUSSION}

In this study, we have reviewed the types of cerebral insult detected in the surviving twin after IUFD in MC pregnancies. Nine cases had radiologic evidence of cerebral abnormalities to the surviving twin. Of those, 4 had significant findings of cerebral ischemia and infarct and 5 had minor findings. In all 9 cases, fetal neurosonographic findings were initially normal (2 had Doppler evaluation suggesting fetal anemia). T2 MR imaging was more sensitive to cerebral findings, and the most sensitive examination was DWI (3 patients with recent ischemic findings on DWI had initially normal T2 MR imaging findings).

Monochorionic twin pregnancies complicated by the death of 1 fetus are associated with substantial mortality in the survivor, with a high risk of developing hypoxic-ischemic damage to the brain. ${ }^{3}$ This has also been published in cases of selective termination either by RFA or bipolar coagulation. ${ }^{14,15}$ The diagnosis of brain abnormalities is performed routinely by fetal neurosonography. Initial findings of brain abnormalities might include intracranial hemorrhage, cerebral necrosis, and cystic lesions. However, the sensitivity of US in the earlier phases of brain damage is poor. ${ }^{16}$ Furthermore, due to the nature of this pathology, the sonographic findings are evolving, and the best timing to detect these pathologies has not been established. Brain damage of monochorionic twins has been diagnosed by prenatal MR imaging, ${ }^{17}$ which might be more sensitive for earlier abnormalities, such as the development of vasogenic edema and initial cystic necrosis. ${ }^{18}$ An additional advantage of prenatal MR imaging over sonography seems to be the capability of MR imaging to detect cortical lesions, which are close to the skull bones. However, even MR imaging usually detects these brain lesions only 2 weeks after the death of the co-twin. ${ }^{17}$ In an animal model of perinatal asphyxia by umbilical cord clamping in the Macaca nemestrina, T2 MR imaging findings only appeared 2 weeks after the injury. ${ }^{19}$

In recent years, clinical applications of DWI in postnatal life have confirmed repeatedly that cerebral ischemic lesions are detected earlier, compared with using conventional T2-weighted imaging. ${ }^{20}$ Moreover, it has been demonstrated in animal models that the ADC value decreases within a few minutes from induction of ischemia because diffusion of water molecules within the tissue is hindered by the swelling of cells and restriction of extracellular space, which are associated with the development of cytotoxic edema. ${ }^{21,22}$ The same mechanism was shown both in animals and human fetuses. ${ }^{23,24}$

A recent case report ${ }^{13}$ has demonstrated that DWI can detect focal ischemic lesions in the survivor of a monochorionic twin pregnancy within the first 1-2 days after the death of the co-twin. In our study, we have demonstrated that MR imaging, when per- formed within a week after the death of the co-twin, can detect recent cerebral infarcts. This is seen even in the absence of sonographic or conventional MR imaging findings (cases A.2, B.1, and C.1).

In our study, early manifestations of cerebral ischemia in monochorionic twin pregnancies were better diagnosed with MR imaging, especially with DWI. Early diagnosis of cerebral ischemic lesions might influence the consultation, parents' decisions, and management of the affected pregnancy. Because a large part of interventions in monochorionic pregnancies are performed at 22-24 weeks of gestation, any brain abnormality related to the procedure will be evident by sonography or T2 MR imaging only when the fetus is considered viable (after 24 weeks' gestation). Therefore, early diagnosis of postprocedural severe brain abnormalities is specifically important when fetal death occurs shortly before delivery (otherwise, patients should wait at least 2 weeks, relying on fetal sonography and T2 MR imaging) or when reaching a strict gestational age limit for termination of pregnancy, whenever patients choose to do so. DWI results can contribute to the decision in such a short period. However, making crucial decisions (ie, pregnancy termination) on the basis of DWI alone could be very difficult because experience in this field is at a very early stage. Our opinion is that termination of pregnancy should be discussed on the basis of DWI results only in cases of clear large cerebral ischemic lesions and not in cases of subtle findings such as germinal matrix or basal ganglia bleeding.

The strength of our study is that the timing of fetal death of all 9 cases (On-line Table) can be narrowed to 1-6 days before the MR imaging. The importance of DWI in such cases is the ability to counsel patients regarding brain ischemic lesions and their consequences shortly after the death of the co-twin and to reassure patients when no abnormality is seen on DWI.

Unfortunately, most pregnant women with abnormal findings in our population elected to terminate the pregnancy following the death of the co-twin, even in the absence of neither recent nor older brain infarcts. Therefore, the limitation of our study is the inability to evaluate the true association between findings on DWI, later brain disruption, and neurodevelopment abnormalities. Therefore, patients should be encouraged, at this point, not to rely on DWI and to await, whenever possible, later results from conventional T2 MR imaging. This is specifically important when DWI shows minor abnormalities with uncertain clinical correlation. DWI of the fetal brain should also be evaluated in larger clinical studies with ongoing pregnancies before adopting it as a formal work-up in cases of monochorionic twin death.

\section{REFERENCES}

1. Hillman SC, Morris RK, Kilby MD. Co-twin prognosis after single fetal death: a systematic review and meta-analysis. Obstet Gynecol 2011;118:928-40

2. Okamura K, Murotsuki J, Tanigawara S, et al. Funipuncture for evaluation of hematologic and coagulation indices in the surviving twin following co-twin's death. Obstet Gynecol 1994;83:975-78

3. Fusi L, McParland P, Fisk N, et al. Acute twin-twin transfusion: a possible mechanism for brain-damaged survivors after intrauterine death of a monochorionic twin. Obstet Gynecol 1991;78:517-20

4. Bajoria R, Wee LY, Anwar S, et al. Outcome of twin pregnancies complicated by single intrauterine death in relation to vascular 
anatomy of the monochorionic placenta. Hum Reprod 1999;14:2124-30

5. Bajoria R, Kingdom J. The case for routine determination of chorionicity and zygosity in multiple pregnancy. Prenat Diagn 1997;17:1207-25

6. Simonazzi G, Segata M, Ghi T, et al. Accurate neurosonographic prediction of brain injury in the surviving fetus after the death of a monochorionic cotwin. Ultrasound Obstet Gynecol 2006;27:517-21

7. Jelin $\mathrm{AC}$, Norton ME, Bartha AI, et al. Intracranial magnetic resonance imaging findings in the surviving fetus after spontaneous monochorionic cotwin demise. Am J Obstet Gynecol 2008;199:398.e1-5

8. Kim DH, Chung S, Vigneron DB, et al. Diffusion-weighted imaging of the fetal brain in vivo. Magn Reson Med 2008;59:216-20

9. Baldoli C, Righini A, Parazzini C, et al. Demonstration of acute ischemic lesions in the fetal brain by diffusion magnetic resonance imaging. Ann Neurol 2002;52:243-46

10. Tarui T, Khwaja OS, Estroff JA, et al. Fetal MR imaging evidence of prolonged apparent diffusion coefficient decrease in fetal death. AJNR Am J Neuroradiol 2011;32:E126-28

11. Righini A, Bianchini E, Parazzini C, et al. Apparent diffusion coefficient determination in normal fetal brain: a prenatal MR imaging study. AJNR Am J Neuroradiol 2003;24:799-804

12. Glenn OA, Quiroz EM, Berman JI, et al. Diffusion-weighted imaging in fetuses with unilateral cortical malformations and callosal agenesis. AJNR Am J Neuroradiol 2010;31:1100-02

13. Righini A, Kustermann A, Parazzini C, et al. Diffusion-weighted magnetic resonance imaging of acute hypoxic-ischemic cerebral lesions in the survivor of a monochorionic twin pregnancy: case report. Ultrasound Obstet Gynecol 2007;29:453-56

14. Paramasivam G, Wimalasundera R, Wiechec M, et al. Radiofrequency ablation for selective reduction in complex monochorionic pregnancies. BJOG 2010;117:1294-98

15. O'Donoghue K, Rutherford MA, Engineer N, et al. Transfusional fetal complications after single intrauterine death in monochorionic multiple pregnancy are reduced but not prevented by vascular occlusion. BJOG 2009;116:804-12

16. Fichera A, Zambolo C, Accorsi P, et al. Perinatal outcome and neurological follow up of the cotwins in twin pregnancies complicated by single intrauterine death. Eur J Obstet Gynecol Reprod Biol 2009;147:37-40

17. Righini A, Salmona S, Bianchini E, et al. Prenatal magnetic resonance imaging evaluation of ischemic brain lesions in the survivors of monochorionic twin pregnancies: report of 3 cases. J Comput Assist Tomogr 2004;28:87-92

18. Garel C, Delezoide AL, Elmaleh-Berges M, et al. Contribution of fetal MR imaging in the evaluation of cerebral ischemic lesions. AJNR Am J Neuroradiol 2004;25:1563-68

19. Juul SE, Aylward E, Richards T, et al. Prenatal cord clamping in newborn Macaca nemestrina: a model of perinatal asphyxia. Dev Neurosci 2007;29:311-20

20. Provenzale JM, Sorensen AG. Diffusion-weighted MR imaging in acute stroke: theoretic considerations and clinical applications. AJR Am J Roentgenol 1999;173:1459-67

21. Müller TB, Haraldseth O, Jones RA, et al. Combined perfusion and diffusion-weighted magnetic resonance imaging in a rat model of reversible middle cerebral artery occlusion. Stroke 1995;26:451-57

22. van der Toorn A, Syková E, Dijkhuizen RM, et al. Dynamic changes in water ADC, energy metabolism, extracellular space volume, and tortuosity in neonatal rat brain during global ischemia. Magn Reson Med 1996;36:52-60

23. Drobyshevsky A, Derrick M, Prasad PV, et al. Fetal brain magnetic resonance imaging response acutely to hypoxia-ischemia predicts postnatal outcome. Ann Neurol 2007;61:307-14

24. Drobyshevsky A, Derrick M, Wyrwicz AM, et al. White matter injury correlates with hypertonia in an animal model of cerebral palsy. J Cereb Blood Flow Metab 2007;27:270-81 